\title{
An application of the $\tau$ scale of taste: \\ Interaction among the four qualities of taste'
}

TAROW INDOW

KEIO UNIVERSITY, TOKYO, JAPAN

\begin{abstract}
A series of 10 standard solutions, spaced equidistant on the $\tau$ scale, were prepared for each of the basic tastes, sweet, bitter, sour, and salty. In addition, four other concentrations of each basic taste, plus 96 compound solutions of two different solutes, were prepared as test stimuli. The Ss, four specialists in taste testing and four laymen, were asked to specify the taste of each test stimulus by matching it with the standard series. In this way, both the precision of the matching procedure and the enhancement or masking of one taste by another were measured in terms of $\tau$, which is a nearly logarithmic function of concentration. The average standard deviations of the matchings among the Ss were in the order of 0.3 to 0.5 units on the $\tau$ scale, which corresponds to a value between 1 and 2 decilogs of concentration. Among the interactions observed was the mutual masking of sucrose and quinine sulfate.
\end{abstract}

The present study concerns the use of the $\tau$ scale (Indow, 1966) to specify the taste of given test solutions. One purpose is to study the precision with which a test solution is allocated by Ss to a point on the $\tau$ scale. Another purpose is to explore the usefulness of the $\tau$ scale in a study of the interactions among taste qualities. Instead of ordinary foodstuffs, aqueous solutions of two solutes, e.g., sucrose and sodium chloride, were used as test solutions, and the taste of these solutions was studied as a function of the concentrations of the constituents.

\section{THE $\tau$ SCALE}

When expressed as functions of concentrations of solutions $\phi$, representing the four qualities of taste, the set of scales $\tau$ obtained in the previous study are represented, with sufficient accuracy, by the formulae:

$$
\begin{array}{lll}
\text { Sucrose } & \tau_{A}=2.65 \log \left(\phi_{A} / 0.48\right) \\
\text { Quinine sulfate } & \tau_{B}=2.35 \log \left(\phi_{B} / 0.00011\right) \\
\text { Tartaric acid } & \tau_{C}=2.27 \log \left(\phi_{C} / 0.0010\right) \\
\text { Sodium chloride } & \tau_{D}=2.83 \log \left(\phi_{D} / 0.056\right)
\end{array}
$$

where $\phi$ is given in terms of gram solute per $100 \mathrm{cc}$ of distilled water.

The basic procedure used to create the $\tau$ scale may be summarized as follows. A pair of solutions was presented and the $\mathrm{S}$ was asked to assess the difference in strength of taste between the two. The pairs consisted either of solutions of different concentration of the same solute (homoqualitative) or of solutions of different solutes (heteroqualitative). Consequently, the same value of $\tau$ represents the same strength of taste irrespective of the qualities. The unit of $\tau$ was defined as the subjective strength of a solution containing $1 \mathrm{~g}$ of sucrose per $100 \mathrm{cc}$ of distilled water, and $\tau$ was defined as zero at the level corresponding to the stimulus threshold of each taste quality (Pfaffmann, 1959). The S judged the difference between a pair of solutions and announced his judgment in terms of the categories: not different, slightly, considerably, or extremely different. These plus the direction of the difference provided seven categories in all. The category judgments of apparent difference were treated by a model whose goodness of fit was tested from various angles in the process of scaling. The most basic assumption of the model was the bivariate-normal distribution assumed between the core taste component and the criterion of each individual $S$. The assumption plays the same role as the assumption of a normal distribution of the "discriminal process" in Thurstone's model for paired comparisons (Thurstone, 1927; Torgerson, 1958). Sirce large stimulus differences were not used, there were vacant cells in the difference matrix. The final result was obtained by processing the incomplete comparison matrix by the method developed by Gulliksen (1956).

A problem arises about how to interpret the value of $\tau$. Because of the prothetic nature of the taste continua (Stevens, $1957,1961)$, the subjective impression of ratio in strength of taste between two solutions, $a$ and $b$, would not be given by the ratio $\tau_{\mathrm{a}} / \tau_{\mathrm{b}}$. When asked to give the ratio, the $\mathrm{S}$ would assign to the pair a value that differs in a nonlinear manner from $\tau_{\mathbf{a}} / \tau_{\mathbf{b}}$. As a matter of fact, in each of the four qualities of taste, $\tau$ was found to be a logarithmic function of the gust scale which was created on the basis of ratio judgments (Lewis, 1948; Beebe-Center, 1949). It is noteworthy that BeebeCenter proposed to use as a measuring device a standard series of solutions that were spaced equally in terms of log gust.

When a scale on a prothetic continuum is based on a model that makes use of small differences and their distributions, the typical outcome is a scale that is a logarithmic function of the stimulus. The $\tau$ scale fulfills this expectation, and it may therefore be classed as a discriminability scale, or a confusion scale. Scales obtained by asking $S$ to match numbers to stimuli in a manner that preserves ratios result in so-called magnitude scales, which are power functions of the stimulus. The work underlying the gust scale produced approximate power functions with exponents near 1.0. Later experiments have given exponents of about 1.3 for sucrose and salt (Stevens, 1961, 1968).

Stimuli

\section{MATCHING EXPERIMENT}

The task was to compare test stimuli with a standard series of 10 solutions for each of the four qualities of taste (Table 1). The four standard series were graphically determined from the results of the previous experiment so as to divide the $\tau$ scales into nine segments of constant width. Hence in Table 1 the stimulus spacing is approximately but not precisely logarithmic. Table 2 gives the 16 basic solutions of which combinations were used as test stimuli. For convenience, solutions of sucrose, quinine sulfate, tartaric acid, and sodium chloride will be denoted by $\mathrm{A}, \mathrm{B}, \mathrm{C}$, and $\mathrm{D}$, respectively, for the standard series and by $a, b, c$, and $d$, respectively, for the basic solutions for test stimuli. Hence, for example, $A_{3}$ represents the third solution of standard series $A$ : a solution of $3 \mathrm{~g}$ of sucrose per $100 \mathrm{cc}$ of distilled water, which defines a sweet taste of 2.0 on the scale $\tau_{\mathrm{A}}$. Similarly, $\mathrm{a}_{2}$ represents a test solution, $4 \mathrm{~g}$ of sucrose per $100 \mathrm{cc}$ of distilled water having a sweet taste of 2.4 on the scale $\tau_{\mathrm{A}}$; and $\mathrm{a}_{2} \mathrm{~b}_{3}$ represents a test solution consisting of $4 \mathrm{~g}$ sucrose and $0.004 \mathrm{~g}$ 
Table 1

Concentrations $(\phi)$ of Standard Series in Terms of Psychological Scale $(\tau)$ and of Grams Solute per $100 \mathrm{cc}$ of Distilled Water

\begin{tabular}{|c|c|c|c|c|c|}
\hline Series & $\tau$ & $\begin{array}{c}\text { A } \\
\text { Sweet } \\
\text { sucrose }\end{array}$ & $\begin{array}{c}\text { B } \\
\text { Bitter } \\
\text { quinine sulfate } \\
\end{array}$ & $\begin{array}{c}\text { C } \\
\text { Sour } \\
\text { tartaric acid } \\
\end{array}$ & $\begin{array}{c}\text { D } \\
\text { Salty } \\
\text { sodium chloride }\end{array}$ \\
\hline 1 & 1.0 & 1.0 & 0.00022 & 0.0025 & 0.12 \\
\hline 2 & 1.5 & 1.9 & 0.00048 & 0.0049 & 0.19 \\
\hline 3 & 2.0 & 3.0 & 0.00090 & 0.0090 & 0.30 \\
\hline 4 & 2.5 & 4.3 & 0.00150 & 0.0155 & 0.49 \\
\hline 5 & 3.0 & 6.4 & 0.00230 & 0.0260 & 0.76 \\
\hline 6 & 3.5 & 9.0 & 0.00370 & 0.0430 & 1.15 \\
\hline 7 & 4.0 & 14.0 & 0.00580 & 0.0700 & 1.60 \\
\hline 8 & 4.5 & 22.5 & 0.00940 & 0.1100 & 2.40 \\
\hline 9 & 5.0 & 34.0 & 0.01500 & 0.1800 & 3.60 \\
\hline 10 & 5.5 & 78.0 & 0.02450 & 0.2900 & 5.00 \\
\hline
\end{tabular}

of quinine sulfate per $100 \mathrm{cc}$ of distilled water. Thus the test stimuli consisted of 16 unmixed basic solutions and of 96 compound solutions. A compound test solution was always a mixture of two solutes of the concentrations given in Table 2 per $100 \mathrm{cc}$ of distilled water. All solutions were maintained at room temperature ( $20 \mathrm{deg} \mathrm{C}, 60 \%$ humidity).

\section{Subjects}

The Ss consisted of two groups, four specialists in taste testing and four nonspecialists. In each group, two of the four were the Ss who had participated in the previous experiment. Three out of the eight Ss were male.

\section{Procedure}

The experiment was conducted in a specially designed room of the Food Research Laboratory under the same conditions as in the previous experiment. Solutions, both the standard series and test stimuli, were presented in 40-cc glasses and, whenever a solution was used up, the $S$ could order another glass of the solution. In a given session, only test stimuli related to a particular pair of solutes (e.g., a and b) were used, and only two standard series of the respective solutes (e.g., A and $B$ ) were presented.

Gregson (1966c) reported that near-threshold mixtures ad sometimes elicit sour and bitter sensations instead of sensations of sweetness and saltiness. In a few cases, some Ss in the present experiment noticed a sensation different from the tastes of the components in compound solutions. No attempt was made, however, to pursue phenomena of this kind.

Two standard series of stimuli were presented, each in the order of intensity, in front of S. In the beginning, the $S$ tasted each glass of the standard series in order to familiarize himself with the series. The $S$ could thereby quickly narrow the range on the standard series with which a given test stimulus was to be compared. He matched the test solution with one of the standard solutions by successively tasting, rinsing, tasting, rinsing, etc. The $S$ took solutions of the standard series, one by one, to compare with the test solution until he gave it an

Table 2

Concentrations $(\phi)$ of Basic Test Stimuli in Terms of Psychological Scale $(\tau)$ and of Grams Solute per $100 \mathrm{cc}$ of Distilled Water

\begin{tabular}{|c|c|c|c|c|c|c|c|c|}
\hline \multicolumn{3}{|c|}{$\begin{array}{r}\text { A } \\
\text { Sweet } \\
\text { sucrose }\end{array}$} & \multicolumn{2}{|c|}{$\begin{array}{c}\text { B } \\
\text { Bitter } \\
\text { quinine sulfate }\end{array}$} & \multicolumn{2}{|c|}{$\begin{array}{c}\text { C } \\
\text { Sour } \\
\text { tartaric acid }\end{array}$} & \multicolumn{2}{|c|}{$\begin{array}{l}\text { D } \\
\text { Salty } \\
\text { sodium chloride }\end{array}$} \\
\hline & $\tau$ & $\phi$ & $\tau$ & $\phi$ & $\tau$ & $\phi$ & $\tau$ & $\phi$ \\
\hline 1 & 1.6 & 2.1 & 1.3 & 0.00036 & 1.9 & 0.008 & 1.4 & 0.17 \\
\hline 2 & 2.4 & 4.0 & 2.4 & 0.00140 & 2.7 & 0.019 & 2.6 & 0.52 \\
\hline 3 & 3.7 & 11.0 & 3.1 & 0.00400 & 3.4 & 0.038 & 3.6 & 1.25 \\
\hline 4 & 4.3 & 18.0 & 4.6 & 0.01100 & 4.7 & 0.114 & 4.9 & 3.40 \\
\hline
\end{tabular}
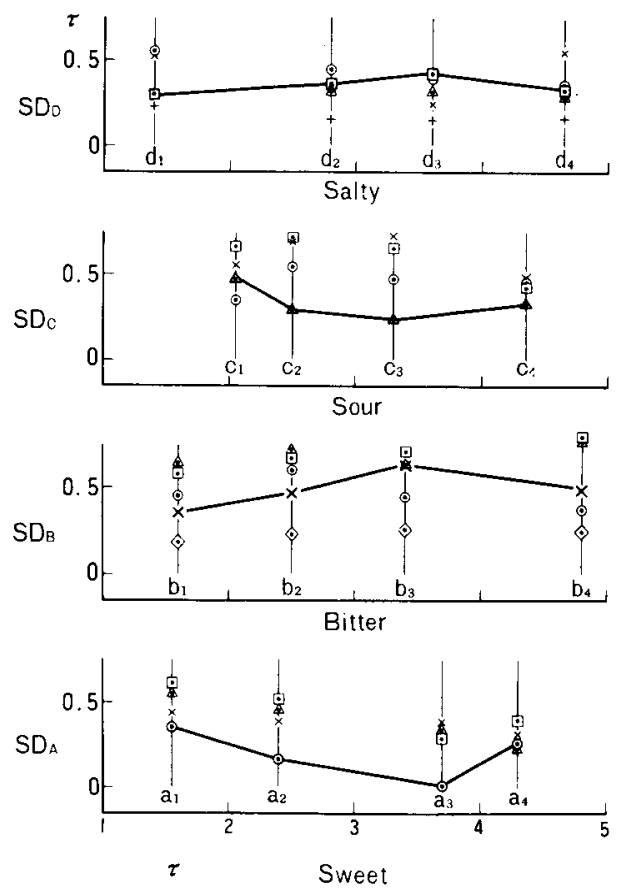

Fig. 1. Standard deviations for the matching of test stimuli with standard taste series. Interindividual SDs (average) for compount solutions are given by various symbols: circle for ax, chi for bx, triangle for $\mathrm{cx}$, and square for $\mathrm{dx}$ where $\mathrm{x}$ denotes any of the three remaining solutes. Symbols connected by lines represent interindividual SDs for unmixed basic solutions. Intraindividual SDs (average) for compound solutions bd are given by diamonds and crosses for matchings with the standard series $B$ and $D$, respectively.

assessment, e.g., $X_{A}=4.2, X_{B}=2.6$ for a solution $a b$, where $X$ represents the position in the respective standard series. The $S$ was encouraged to interpolate, if necessary, within the interval between adjacent standard solutions. About 20 test solutions were judged in a session, which lasted $1 \frac{1}{2} \mathrm{~h}$ including intermissions. The test solutions comprised 16 unmixed basic solutions and 96 combined solutions, for a total of 112 . In addition, 8 out of 32 combined solutions of the type bd were presented twice in two different sessions for the purpose of examining the reliability of judgment. Thus the total number of presentations to each $\mathrm{S}$ was 120 , which required six sessions. Within a session, test solutions were presented in irregular order, a different order for each $\mathrm{S}$.

\section{VARIABILITY}

Although $\mathrm{S}$ reported his judgments in terms of position within a standard series, the results will be stated in terms of $\tau$. Let us first consider precision. In Fig. 1 are shown standard deviations (SD) of judgments, interindividual and intraindividual. Interindividual SDs for test solutions of unmixed basic solutions are given by the symbols connected by lines. Averages of four interindividual SDs for combined solutions are plotted in the same figures. For example, the chi, the symbol for $b$, on the $a_{1}$-line in the bottom figure shows the average of four SDs for $a_{1} b_{1}, a_{1} b_{2}, a_{1} b_{3}$, and $a_{1} b_{4}$, each representing the scatter of assessments of sweetness by the eight Ss. Similarly, the chi on the $d_{1}$-line in the top figure shows the average of four SDs for $b_{1} d_{1}, b_{2} d_{1}, b_{3} d_{1}$, and $b_{4} d_{1}$, each representing the scatter of assessments of saltiness by the eight Ss. In the latter example, $b_{1} d_{1}$ and $b_{4} d_{1}$ are the cases in which the test solution was presented twice. These repeated judgments by the same $S$ had been averaged before taking the corresponding interindividual SD. When judgments 
were repeated, intraindividual SDs were also obtained. They are shown by crosses and diamonds in the top and third figures. For example, the cross on $d_{1}$-line in the top figure shows the average of eight intraindividual SDs for $b_{1} d_{1}$ and also eight SDs for $b_{4} d_{1}$, each representing the scatter of two assessments of saltiness repeated by the same $S$.

The following facts seem noteworthy. Both inter- and intraindividual SDs remain approximately at a constant level of $\tau$, irrespective of the concentration of the solution to be measured. Furthermore, the value of the interindividual SDs is practically the same for the four qualities of taste: about 0.3-0.5 in units of $\tau$, or between 1 and 2 decilogs of concentration. (One unit of $\tau$ equals approximately 4 decilogs.) Since $\tau$ is approximately a logarithmic function of stimulus concentration, the constancy of the SD, in units of $\tau$, may be regarded as evidence for Weber's law, i.e., the relative variability is constant. The relative variability is roughly the same for all the tastes. For A (sweet) and C (sour), the interindividual SDs are smaller for unmixed basic solutions than for compound solutions. That is not true for B (bitter) and D (salty), however. As would be expected, the intraindividual SDs turned out somewhat smaller than interindividual SDs. The intraindividual SD is a decilog or slightly less, which accords well with numerous measures of the Weber fraction for taste.

\section{MATCHING RESULTS}

The arithmetic means of the $\tau$ values of the matches made by the eight Ss are given in the six plots of Fig. 2. For example, in the first plot, a point of intersection of two straight lines $a_{i}$ and $b_{j}$ indicates a compound sweet-bitter test solution $a_{i} b_{j}$ and the circle and the chi near the intersecting point represent the obtained sweetness and bitterness of the solution in terms of $r$. The sweetness of the basic test solutions $a_{i}$ are represented by circles on the lowest horizontal line and the bitterness of the basic test solutions $b_{j}$ by chi's on the leftmost vertical line. Other plots may be read in a similar way.

In theory, the obtained values for the unmixed basic solutions should fall on the intersecting points on the lowest and leftmost lines, i.e., on the appropriate zero coordinate. Since each point is the mean of eight (in some cases nine) specifications having an SD of the order of 0.3 to 0.4 each, the SD of a point would be about 0.15 . Hence, in the case of unmixed basic solutions, the deviation of a point in the vertical or horizontal direction from its corresponding intersecting point may be regarded as statistically significant if it exceeds 0.4 in terms of $\tau$. All the deviations for the unmixed solutions. were well within the limits of random error. However, quinine sulfate $b$ gave systematically larger values of $\tau$ than expected, although no one of the deviations was significant in the statistical sense. It is possible that error may have occurred in the process of preparing the test solutions $b$ and/or the standard solutions B, since an extremely small amount of solute had to be dealt with in the preparation of the bitter solutions.

\section{INTERACTIONS}

The problem of the interaction between pairs of taste qualities has been dealt with in the series of reports by Pangborn and her associates (Pangborn, 1960, 1961, 1962, 1963, 1964; Pangborn \& Chrisp, 1964; Pangborn, Ough, \& Chrisp, 1964; Pangborn \& Trabue, 1964); by Kamenetzky and Pilgrim (1958); and in an entirely different approach by Békésy (1964). The picture is not simple, and the same may be said of the present results. The interactions are given in Fig. 2 by deviations, vertical or horizontal, of the points representing compound solutions. As stated before, a deviation exceeding 0.4 in terms of $\tau$ may be regarded as significant, although this criterion may need to be modified to take account of the deviation of the unmixed basic solution, as in the case of $b$.
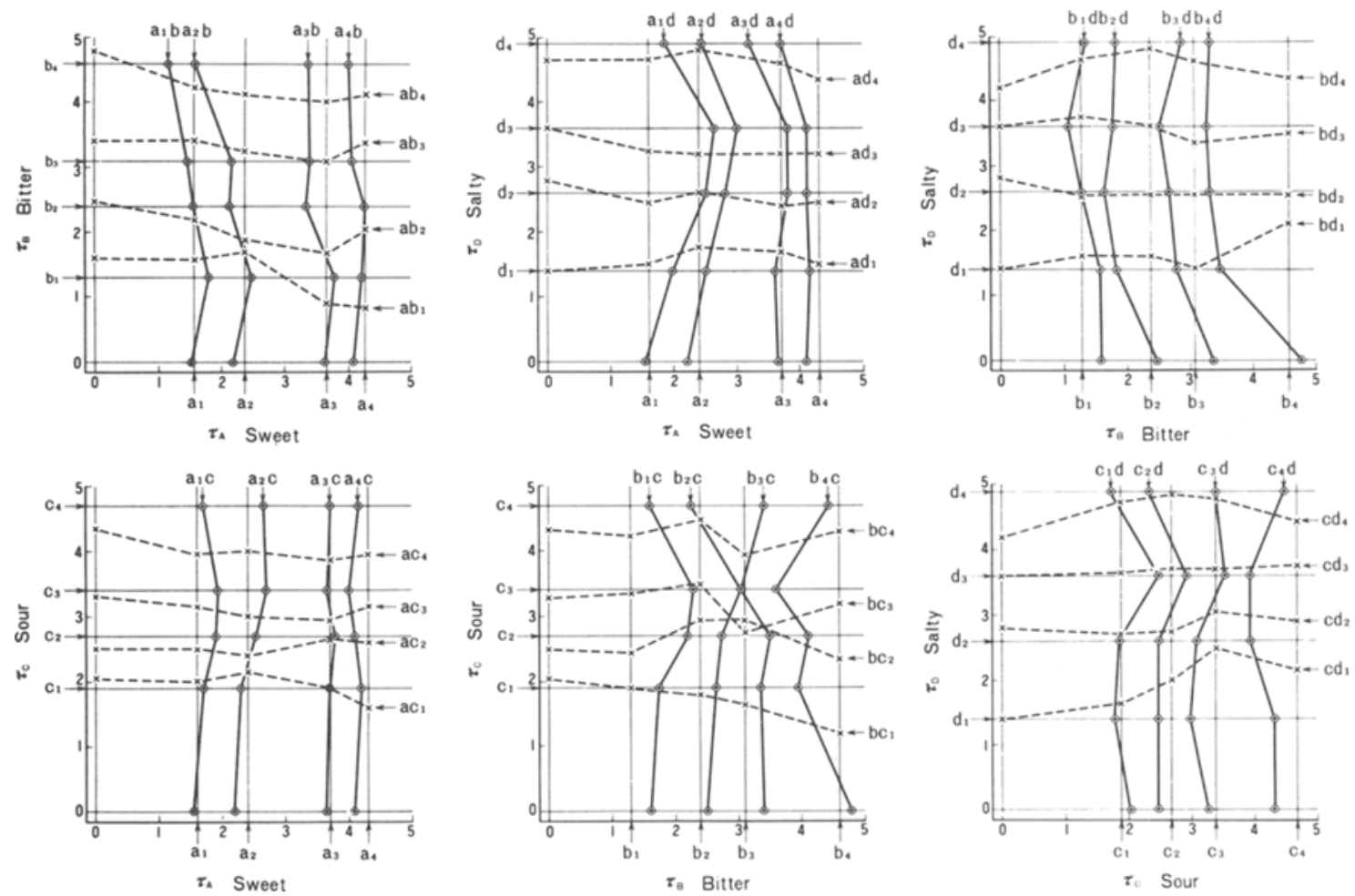

Fig. 2. Interactions between the two qualities of taste produced by two solutes in compound solutions. Circles and chi's represent mean positions of test stimuli located as a result of matching, on the respective $\tau$ scales. Concentrations in terms of $\tau$ of the respective solutes in compound solutions are given by the straight lines. 
Since the SDs of the respective points are about 0.15 , the SD for difference would be on the order of 0.26 . Hence, according to the latter criterion, a deviation defined from the taste of the corresponding unmixed solution will be significant when it exceeds 0.7 in terms of $\tau$. In the summary that follows, however, emphasis is laid less on the absolute size of the deviation than on the systematic pattern of the deviations.

\section{Change of Sweetness of Sucrose a}

1.1 A slight but consistent masking effect was observed at $a_{1} b$ to $a_{4} b$ when quinine sulfate was at the higher levels, $b_{3}$ and $b_{4}$.

1.2 The masking due to sodium chloride was observed only at $\mathrm{a}_{3} \mathrm{~d}_{4}$ and $\mathrm{a}_{4} \mathrm{~d}_{4}$. The change was of medium size.

1.3 The most marked enhancement took place with the lower levels of sucrose, $a_{1}$ and $a_{2}$, at the intermediate levels of sodium chloride, $d_{2}$ and $d_{3}$.

1.4 A slight enhancement was observed with $a_{1} b$ to $a_{3} b$ at the lowest level of quinine sulfate $b_{1}$.

1.5 A slight enhancement was also observed with $a_{1} c$ and $a_{2} c$ at the higher levels of tartaric acid $c$.

\section{Change of Bitterness of Quinine Sulfate $b$}

2.1 A large and consistent masking effect was observed at $a b_{1}$ to $a b_{4}$ when sucrose was at the higher levels, $a_{2}$ to $a_{4}$.

2.2 A large masking was also observed at $b_{2} d$ to $b_{4} d$ for all the levels of sodium quinine $d$.

2.3 Moderate masking took place with $b_{3}$ and $b_{4}$ at a particular level of tartaric acid $c_{3}$.

2.4 A moderate enhancement was observed at $b_{1} c$ and $b_{2} c$ when tartaric acid was at the intermediate levels, $c_{2}$ and $c_{3}$. No other enhancement was observed with $b$.

\section{Change of Sourness of Tartaric Acid $c$}

3.1 A slight but consistent masking effect was observed at $\mathrm{ac}_{3}$ and $\mathrm{ac}_{4}$ for all the levels of sucrose $\mathrm{a}$.

3.2 A slight but consistent masking effect was also observed at $b c_{1}, b c_{3}$, and $b c_{4}$ when quinine sulfate was at the higher levels, $b_{3}$ and $b_{4}$.

3.3 Considerable enhancement took place with $c_{1}$ to $c_{3}$ at a particular level of sodium chloride $d_{3}$. No other enhancement was observed with $c$.

\section{Change of Saltiness of Sodium Chloride d}

4.1 The taste of sodium chloride was fairly independent in the presence of other solutes.

4.2 A masking, slight but consistent, was observed only at $\mathrm{ad}_{3}$ for all the levels of sucrose $\mathrm{a}$.

4.3 Considerable enhancement took place only with $d_{1}$ at the higher levels of tartaric acid, $c_{3}$ and $c_{4}$.

\section{DISCUSSION}

The interaction among the four qualities of taste can be approached in various ways. Gregson (1966a, b; Gregson \& Russell, 1966) applied multidimensional scaling to this problem. Békésy (1964) developed the duplexity theory of taste with a special method. He presented two different taste stimuli, one to the left and the other to the right side of the tongue and observed whether they added together to a sensation in the middle of the tongue or stayed separated. The interaction occurred only within the pairs of tastes, sweet and bitter, sour and salty. In the present study, however, interactions of different kinds, inhibition and/or enhancement, were observed not only within these pairs but also within the pairs, sweet and salty, bitter and sour.

As to the findings about enhancement and masking enumerated above, some agree with results obtained by other investigators and some do not. The finding 2.1 , the masking of bitter by sucrose, seems to correspond to the frequently observed fact that sucrose decreases the bitterness of caffeine. The interaction between sucrose and sodium chloride has been studied most thoroughly in the past with aqueous solutions (Beebe-Center et al, 1959; Pangborn, 1962) and even with foodstuffs (Pangborn \& Trabue, 1964; Pangborn \& Chrisp, 1964; Pangborn et al, 1964). The findings 1.2 and 1.3 are qualitatively of the same pattern of interaction as reported in those studies. The masking of saltiness by sucrose has also been reported. This masking was not clear in the present experiment except for the finding 4.2. The mutual masking effect between sucrose and acid has been reported, both in solutions (Pangborn, 1961, 1964) and in foodstuffs (Pangborn \& Chrisp, 1964; Pangborn \& Trabue, 1964; Pangborn et al, 1964). Though the masking of the sour taste of tartaric acid by sucrose was noted in 3.1, no appreciable masking the other way around was found in the present study.

A comment about the use of the $\tau$ scale to investigate taste interaction seems in order. By the use of the $\tau$ scale, changes in different qualities of taste become comparable with each other. The comparability is analogous to that achieved by the use of sensation level in hearing (decibels above threshold).

In the use of the matching procedure, there is a tacit assumption that the two qualities of taste in a compound solution may interact with each other, but they do not affect the tastes of the standard series of solutions. For example, the sweetness of component a may affect the bitterness of $b$ in a compound solution ab but it affects neither the taste of A nor the taste of $B$ of the respective standard series. The constituents of the compound solutions stimulated the tongue simultaneously, but the compound solution and single solution of the standard series were tasted in succession, with the mouth rinsed in between. The assumption of the independence of two tastes that are given in succession, with rinsing in between, would seem necessary for all kinds of taste experiments. The same assumption was implicitly made in the procedure by which the $\tau$ scale was originally constructed.

\section{REFERENCES}

BEEBE-CENTER, J. G. Standards for use of the gust scale. Journal of Psychology, 1949, 28, 411-419.

BEEBE-CENTER, J. G., ROGERS, M. S., \& ATKINSON, W. H. Sweetness and saltiness of compound solutions of sucrose and $\mathrm{NaCl}$ as a function of concentration of solutes. Journal of Experimental Psychology, 1959, 57, 231-234.

BÉKÉSY, G. V. Duplexity theory of taste. Science, 1964, 145, 834-835

GREGSON, R. A. M. Cross-modal matching and four-component taste mixtures, with one component fixed, under two pacing conditions. Perceptual \& Motor Sk ills, 1966a, 23, 183-190.

GREGSON, R. A. M. Theoretical and empirical multi-dimensional scaling of taste mixture matchings. British Journal of Mathematical \& Statistical Psychology, 1966b, 19, 59-75.

GREGSON, R. A. M. Qualitative identification of the taste of weak sucrose-sodium chloride mixture stimuli. Perception \& Psychophysics, $1966 \mathrm{c}, 1,154-156$.

GREGSON, R. A. M., \& RUSSELL, P. N. A comparison of intermodal and intramodal method in the multi-dimensional scaling of three-component taste mixtures. Australian Journal of Psychology, $1966,18,244-254$.

GULLIKSEN, H. A least squares solution for paired comparisons with incomplete data. Psychometrika, 1956, 21, 125-135.

INDOW, T. A general equi-distance scale of the four qualities of taste. Japanese Psychological Research, 1966, 8, 136-150.

KAMENETZKY, J., \& PILGRIM, F. J. The effect of sucrose upon the perceived intensity of salt and bitter. American Psychologist, 1958, 13,420 .

LEWIS, D. R. Psychological scales of taste. Journal of Psychology, 1948, $26,437-446$.

PANGBORN, R. M. Taste interrelationships. Food Research, 1960, 25, 245-256.

PANGBORN, R. M. Taste interrelationships. II. Suprathreshold solutions of sucrose and citric acid. Journal of Food Science, 1961, 26, 648-655.

PANGBORN, R. M. Taste interrelationships, III. Suprathreshold solutions of sucrose and sodium chloride. Journal of Food Science, $1962,27,495-500$. 
PANGBORN, R. M. Relative taste intensities of selected sugars and organic acids. Journal of Food Science, 1963, 28, 726-733.

PANGBORN, R. M. Taste interrelationships of organic acids and selected sugars. In J. M. Leith (Ed.), Quality analysis and composition of foods. Vol. 3: Food science and technology. New York: Gordon and Breach, 1964.

PANGBORN, R. M., \& TRABUE, I. M. Taste interrelationships. V. Sucrose, sodium chloride, and citric acid in Lima bean puree. Journal of Food Science, 1964, 29, 233-240.

PANGBORN, R. M., \& CHRISP, R. B. Taste interrelationships. VI. Sucrose, sodium chloride, and citric acid in canned tomato juice. Journal of Food Science, 1964, 29, 490-498.

PANGBORN, R. M., OUGH, C. S., \& CHRISP, R. B. Taste interrelationships of sucrose, tartaric acid, and caffeine in white table wine. American Journal of Enology \& Viticulture, 1964, 15, 154-161.

PFAFFMANN, C. The sense of taste. In H. W. Magoun (Ed.), Handbook of physiology. Section 1: Neurophysiology. Washington, D.C.: American Physiological Society, 1959. Pp. 507-533.
STEVENS, S. S. On the psychophysical law. Psychological Review, 1957, $64,153-181$

STEVENS, S. S. The psychophysics of sensory function. In W. A. Rosenblith (Ed.), Sensory communication. Cambridge, Mass.: The M.I.T. Press, 1961.

THURSTONE, L. L. A law of comparative judgment. Psychological Review, 1927, 34, 273-286.

TORGERSON, W. S. Theory and method of scaling. New York: Wiley, 1958.

\section{NOTES}

1. The present study was conducted in 1963 by the author's students $M$. Ueda and $H$. Tsuzuki, with the cooperation of S. Ikeda and his staff at the Food Research Laboratory of Ajinomoto Co. Their cooperation is greatly appreciated.

2. Address: Department of Psychology, Keio University, Tokyo, Japan.

(Accepted for publication December 13, 1968.) 